\title{
Bactérias do gênero Aeromonas em carcaças e cortes comerciais de frangos comercializados em Jaboticabal, Estado de São Paulo e comportamento das cepas frente à ação de antimicrobianos
}

\author{
Bacteria of the strain Aeromonas in carcasses and commercial cuts \\ poultry commercialized in Jaboticabal, State of São Paulo and the \\ behavior of strains facing antimicrobian action
}

Darci Lara Perecin Nociti, ${ }^{\star}$ Oswaldo Durival Rossi Júnior, ${ }^{\star \star}$ Luiz Augusto do Amaral, ${ }^{\star \star}$ Antonio Nader Filho**

\begin{abstract}
Resumo
Bactérias do gênero Aeromonas têm sido incriminadas como agentes de infecções tanto intestinal como extra-intestinais, sendo os alimentos de origem animal considerados como importantes veículos na sua transmissão ao homem, particularmente aqueles produtos que entram em contato com a água durante sua obtenção ou preparação. Assim, dadas as limitadas informações na literatura nacional a respeito da presença de aeromonas em carne de aves colhidas no comércio, foram analisadas 30 amostras de carcaças, 30 de coxa/sobrecoxa e 50 peitos de frangos adquiridos em Jaboticabal/SP. A positividade para o gênero foi de $34,54 \%$ do total de amostras analisadas, sendo que em $29,09 \%$ delas (32) foi isolada a espécie $A$. hydrophila e em $23,63 \%$ (26) a $A$. caviae. Apresentaram-se positivas $50 \%$ das amostras de carcaças, $28 \%$ das de peito e $30 \%$ das de coxa com sobrecoxa, com populações mínima de $0,3 \times 10$ e máxima de $2,1 \times 10^{5}$ UFC/grama de amostra. Cerca de $80 \%$ das cepas isoladas foram resistentes à polimixina, carbenicilina, cefoxitina e cefalotina e $49 \%$ a todos os antimicrobianos testados. No isolamento seletivo o ágar vermelho de fenolamido-ampicilina revelou $17,27 \%$ de amostras positivas e o ágar dextrina-ampicilina $30 \%$. Os resultados evidenciam risco ao consumidor, tendo em vista o número significativo de amostras com isolamento positivo e o alto índice de resistência verificado.
\end{abstract}

Palavras-chave: Aeromonas sp.; Aeromonas hydrophila; Aeromonas caviae; carne de aves; antimicrobianos.

\section{Introdução}

Dentre os agentes de toxinfecções veiculados por alimentos, alguns estão bem caracterizados, com patogenia e epidemiologia conhecidas, como o Staphylococcus aureus, Clostridium perfringens e salmonelas de uma forma geral, e outros ditos emergentes, estando neste grupo a Yersinia enterocolitica, a Listeria monocytogenes, Campylobacter sp. e as aeromonas móveis.

De acordo com a $8^{a}$ edição do Bergey's Manual of Systematic Bacteriology (Popoff, 1984), o gênero Aeromonas compreende bactérias anaeróbias facultativas, sendo a maioria delas móveis por flagelo polar. Este gênero é subdividido em dois grupos, um deles móvel e mesófilo que inclui as espécies $A$. hydrophila, $A$. caviae e $A$. sobria e o outro imóvel e psicrofílico que apresenta a espécie $A$. salmonicida com as subespécies salmonicida, achromogenes e masoucida. A A. hydrophilaé considerada a espécie tipo.
A maioria dos estudos da ecologia das gastroenterites determinadas pelas aeromonas têm a preocupação de estabelecer a água como principal veículo de transmissão (Kipperman et al., 1984, Picard e Goullet, 1987, Krovacek et al., 1989, Merino et al., 1995, Handfield et al., 1996) sendo esta considerada, por muitos pesquisadores, como a principal via de contaminação para os alimentos (Agger et al., 1985, Fricker e Tompsett, 1989).

Estudos mais recentes sobre Aeromonas sp., em diferentes alimentos, têm revelado um alto grau de isolamento $e$ alguns investigadores concluem que os alimentos também desempenham papel de importância na transmissão do microrganismo ao homem (Jindal et al., 1993, Rossi Júnior et al., 1996).

Barnhart et al. (1989), através de exames de carcaças colhidas em vários estágios num abatedouro avícola, mostraram que a $A$. hydrophila pode ser rotineiramente isolada

\footnotetext{
* Pós-graduanda do Curso de Medicina Veterinária, Área de Concentração Medicina Veterinária Preventiva, FCAVJ-UNESP.

* Departamento de Medicina Veterinária Preventiva, FCAVJ-UNESP, Rodovia Carlos Tonanni Km 5, 14870-000 Jaboticabal, SP, Brasil.
} 
nos diferentes pontos do fluxograma de abate. Hänninen (1993) confirma a ocorrência da Aeromonas sp. em amostras de frangos e a predominância da espécie $A$. hydrophila, que foi isolada em $62 \%$ das amostras. Chetti et al. (1993) e Cwiková et al. (1993) apresentam dados semelhantes a partir de carcaças obtidas no comércio. Estes autores verificaram a ocorrência de Aeromonassp. em 42\% e 41,4\% de amostras, respectivamente.

Nos últimos anos o consumo de aves tem aumentado de forma acentuada, quer como decorrência da elevação dos preços de outras fontes de proteína animal, quer como conseqüência de uma alteração dos hábitos alimentares da população. Assim, é de interesse que se realizem estudos relativos à população microbiana veiculada por este alimento, visando não só a proteção da saúde do consumidor, como também a sua conservação.

Tendo em vista as considerações apresentadas, principalmente no que diz respeito à possibilidade da transmissão das aeromonas via alimentos, idealizou-se o presente trabalho com os objetivos de verificar a ocorrência do microrganismo em amostras de carcaças e cortes comerciais de frango, adquiridos no comércio varejista de Jaboticabal, $\mathrm{SP}$, verificar o comportamento das cepas isoladas frente à ação de antimicrobianos de uso comum e contribuir com informações relacionadas à metodologia de isolamento a partir de alimentos.

\section{Material e métodos}

Foram analisadas 110 amostras de frangos, representadas por 30 amostras de carcaças, 30 de coxa/sobrecoxa e 50 de peito, obtidas no mercado varejista de Jaboticabal, estado de São Paulo. As amostras eram embaladas na forma comum de venda ao consumidor e transportadas ao laboratório acondicionadas em caixas de isopor contendo blocos de gelo.

No laboratório as amostras foram submetidas à técnica da enxaguadura (Johnston e Tompkin, 1992) e, para tal, as carcaças eram acondicionadas em sacos de polietileno e no conjunto vertidos $300 \mathrm{~mL}$ de caldo tripticase soja (TSB) adicionado de ampicilina na proporção de $10 \mathrm{mg}$ por litro (Abeyta Júnior et al., 1990). Os cortes eram acondicionados como da forma anterior e adicionados de $100 \mathrm{~mL}$ do citado caldo. Todo o conjunto era agitado por um minuto e a solução vertida em frasco tipo Erlenmeyer.

Para a pesquisa de bactérias do gênero Aeromonasa solução de lavagem sofria um enriquecimento a $28^{\circ} \mathrm{C} / 24$ horas, em incubadora para $B O D$, após o que procedia-se ao plaqueamento em ágar vermelho de fenol-amido-ampicilina (Palumbo et al., 1985, Majeed et al., 1990) e ágar dextrinaampicilina (Havelaare Vonk, 1988). Após incubação a $28^{\circ} \mathrm{C} /$ 24 horas eram tomadas até cinco Unidades Formadoras de Colônias (UFCs) sugestivas do gênero, ou seja, UFCs amarelas com halo transparente decorrente da hidrólise do amido ou da dextrina, as quais eram repicadas em tubos com ágar tripticase soja inclinado e, após incubação, semeadas em ágar tríplice açúcar ferro. As culturas que apresentavam reação ácida tanto na base como no bisel, com ou sem formação de gás, eram submetidas às provas da motilidade, oxidase, catalase e resistência ao agente vibriostático 2,4-diamino-6,7-diisopropyl-pteridine (O/129) (Popoff, 1984), sendo consideradas como de Aeromonas $\mathrm{sp}$. as que apresentavam resultados positivos nessas provas.

A caracterização das espécies era realizada seguindo o esquema proposto por Popoff (1984) acrescido de algumas outras provas recomendadas por Buchanan e Palumbo (1985) e Abeyta Júnior et al. (1990), ou seja, produção do indol, hidrólise da esculina e da arginina, descarboxilação da lisina e da ornitina, produção de ácido a partir do inositol, fermentação da salicina, sacarose, manitol e da arabinose e produção de acetoína e gás a partir da glicose.

A contagem do gênero era realizada a partir da solução de enxaguadura ou suas diluições decimais, através da inoculação de $0,1 \mathrm{~mL}$ na superfície de placas contendo ágar vermelho de fenol amido ampicilina. Eram contadas as colônias características, as quais eram em seguida submetidas às provas de confirmação. Para a transformação dos resultados de Unidades Formadoras de Colônias/ mililitro (UFCs $/ \mathrm{mL}$ ) da solução de enxágüe para UFCs $/ g$ de amostra, considerava-se o volume da solução $(300 \mathrm{~mL}$ para as carcaças e $100 \mathrm{~mL}$ para os cortes) como equivalente ao peso da amostra. Com isso obtinha-se, para cada amostra, um fator de conversão. Para discussão dos resultados foram calculadas as médias geométricas e aritméticas.

Foi verificado também o comportamento de 29 cepas de Aeromonas hydrophila e 24 cepas de Aeromonas caviae, frente à ação de antimicrobianos de uso comum, utilizando-se a técnica de Bauer et al. (1966).

\section{Resultados e discussão}

Pelos dados apresentados na Tabela 1 verifica-se a existência de um número significativo de amostras positivas para o gênero Aeromonas, sendo este da ordem de $50 \%$ para as carcaças, $28 \%$ para as de peito e $30 \%$ para as de coxa/sobrecoxa. Estes dados se apresentam bem inferiores aos verificados por Gobat e Jemmi (1993), Barnhart et al. (1989) e Hänninen (1993) que encontraram, respectivamente, $84,4 \%, 98 \%$ e $94 \%$ de amostras positivas, e semelhantes aos verificados por Chetti (1993) e Cwiková et al. (1993), que encontraram $42 \%$ e $41,4 \%$ de amostras positivas, respectivamente, ao analisarem carcaças obtidas no comércio.

Em vista dos resultados obtidos, todos esses autores são unânimes na preocupação dos alimentos citados servirem como veículo na transmissão das aeromonas ao homem. 
Tabela 1: Número de amostras de carcaça e cortes comerciais de frangos obtidos no varejo, positivas para o gênero Aeromonas, em relação ao total analisado

\begin{tabular}{c|c|c}
\hline Amostra & Total analisado & Total positivo \\
\hline Carcaça & 30 & $15(50)^{*}$ \\
Peito & 50 & $14(28)$ \\
Coxa/Sobrecoxa & 30 & $9(30)$ \\
\hline Total & 110 & $38(34,54)$ \\
\hline
\end{tabular}

* Porcentagem de amostras positivas

$\mathrm{Na}$ Tabela 2 verifica-se que as espécies de aeromonas encontradas foram a $A$. hydrophila e a $A$. caviae, sendo a primeira isolada em $32(29,09 \%)$ e a segunda em 26 $(23,63 \%)$ das amostras analisadas. Barnhart et al. (1989) verificaram que a espécie $A$. hydrophila foi a mais prevalente, tendo sido encontrada em $100 \%$ das amostras de carcaças de frangos analisadas. Esta espécie também foi a mais encontrada por Gobat \& Jemmi (1993), que a verificaram em $42,2 \%$ das amostras de carcaças de frango enquanto que a $A$. caviae foi encontrada em $22,2 \%$ delas.

A predominância da espécie $A$. hydrophila nas amostras analisadas vem ao encontro ainda do verificado por outros pesquisadores. Esse microrganismo foi isolado de aves adquiridas no comércio, conservadas sob refrigeração (Palumbo et al., 1989, Barnhart et al., 1989) ou pré-cozidas (Toule e Murphy, 1978), reforçando a afirmativa de que as carnes de frangos podem desempenhar papel de importância na epidemiologia de gastroenterites, tendo em vista que a citada espécie tem sido classificada como a mais importante do gênero em termos de patogenicidade ao homem.

Tabela 2: Número de amostras positivas para cada uma das espécies de Aeromonas identificadas, em relação a cada um dos tipos de amostras estudadas

\begin{tabular}{c|c|cc}
\hline \multirow{2}{*}{ Tipo de amostra } & & \multicolumn{2}{|c}{ Aeromonas sp. } \\
\cline { 3 - 4 } & Total de amostras & A.hydrophila & A.caviae \\
\hline Carcaça & $30 / 15^{1}$ & $11^{2}(36,66)^{3}$ & $13(43,33)$ \\
Peito & $50 / 14 \cdot$ & $13(26,0)$ & $08(16,0)$ \\
Coxa/Sobrecoxa & $30 / 09$ & $08(26,66)$ & $05(16,66)$ \\
\hline Total & $110 / 38$ & $32(29,09)$ & $26(23,63)$ \\
\hline
\end{tabular}

1 Total de amostras analisadas/total de amostras positivas para o gênero.

${ }^{2}$ Número de amostras em que a espécie foi isolada.

${ }_{3}$ Porcentagem em relação ao total analisado.
Com relação às contagens de bactérias do gênero Aeromonas, verifica-se na Tabela 3 que, nas amostras de carcaça, os valores variaram de $2,0 \times 10$ a $1,0 \times 10^{4} \mathrm{UFCs/}$ $\mathrm{mL}$ da solução de enxágüe, sendo a média aritmética de $1,1 \times 10^{3}$ e a média geométrica de $1,5 \times 10^{2}$ e nas amostras

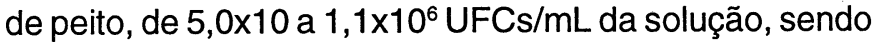
a média aritmética $1,4 \times 10^{5}$ e a média geométrica $5,7 \times 10^{3}$. Os valores médios obtidos nas carcaças, convertidos em UFCs/grama de amostra, revelam populações de $1,7 \times 10^{2}$ e 2,2×10 para as médias aritméticas e geométricas, respectivamente, enquanto que nas amostras de peito, es-

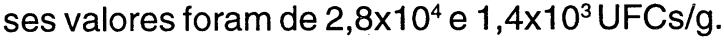

Barnhart et al. (1989) encontraram em carcaças valores de 2,8x10 UFCs/mL na solução de lavagem imediatamente após o resfriamento e $5,8 \times 10^{2}$ UFCs $/ \mathrm{mL}$ na solução de lavagem na fase de pós evisceração.

A falta de mais informações referentes a esta determinação em carne de frangos dificulta uma análise mais profunda, no sentido de se estabelecer a sua real significância. Trabalhos com outros produtos de origem animal já foram realizados e verificadas contagens bem superiores àquelas aqui descritas, da ordem de $3,0 \times 10^{7}, 2,1 \times 10^{7} \mathrm{e}$

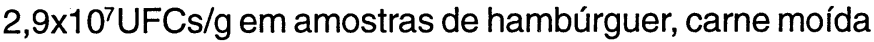
e salsicha, respectivamente (Fathi e Moustafa, 1991) e de $6,0 \times 10^{6} \mathrm{UFCs} / \mathrm{g}$ em amostras de carne moída (Gobat e Jemmi, 1993).

Muito embora o número de células viáveis encontrado no presente estudo tenha sido inferior ao de outros produtos cárneos, não pode ser esquecido que essas bactérias podem se desenvolver durante as fases de distribuição e comercialização onde, na maioria das vezes, as condições de conservação permitem a multiplicação, podendo atingir números preocupantes. Neste aspecto Knochel (1990) lembra o fato de as Aeromonas sp. serem ativas deteriorantes através de eficiente sistema de exoenzimas.

Tabela 3: Valores das populações mínimas e máximas de Aeromonas sp. e médias aritméticas e geométricas, obtidas nas amostras de carcaças e peitos de frango

\begin{tabular}{l|cc|cc}
\hline \multirow{2}{*}{ População } & \multicolumn{2}{|c|}{ Carcaças } & \multicolumn{2}{c}{ Peitos } \\
\cline { 2 - 5 } & UFCs $/ \mathrm{mL}^{1}$ & UFCs/g & UFCs $/ \mathrm{mL}$ & UFCs/g \\
\hline \multirow{2}{*}{ Mínima } & $2,0 \times 10$ & $0,3 \times 10$ & $5,0 \times 10$ & $1,0 \times 10$ \\
Máxima & $1,0 \times 10^{4}$ & $1,5 \times 10^{3}$ & $1,1 \times 10^{6}$ & $2,1 \times 10^{5}$ \\
\hline Média aritmética & $1,1 \times 10^{3}$ & $1,7 \times 10^{2}$ & $1,4 \times 10^{5}$ & $2,8 \times 10^{4}$ \\
Média geométrica & $1,5 \times 10^{2}$ & $2,2 \times 10$ & $5,7 \times 10^{3}$ & $1,4 \times 10^{3}$ \\
\hline
\end{tabular}

1 Dados em unidades formadoras de colônias por mililitro da solução de lavagem.

2 Dados em unidades formadoras de colônias por grama de amostra. 
O comportamento das cepas de aeromonas frente à ação de antimicrobianos pode ser verificado na Tabela 4 . As porcentagens de resistência à ampicilina, polimixina, carbenicilina, cefoxitina e cefalotina foram superiores a $80 \%$ e cerca de $47 \%$ das cepas foram sensíveis à netilmicina e tobramicina e $49 \%$ delas sensíveis à gentamicina. Jindal et al.(1993) encontraram alto índice de resistência a amoxacilina e penicilina em cepas isoladas de fezes humanas e de aves e Hassani et al. (1992) encontraram cepas isoladas da água de consumo da cidade de Marrakech, Marrocos, altamente resistentes a amoxacilina ( $100 \%$ ) e cefalotina ( $73 \%)$. Verifica-se, por estes dados, particularmente no que diz respeito à cefalotina, que os resultados encontrados no presente estudo, ou seja, $83 \%$ de cepas resistentes, são bastante próximos. Por outro lado, Singh e Sanyal (1994), estudando cepas isoladas de casos clínicos de diarréia humana, verificaram baixa resistência a estreptomicina $(2 \%)$, tetraciclina $(2 \%)$ e sulfazotrim $(1,3 \%)$.

O número elevado de cepas isoladas de carne de aves que se apresentaram resistentes aos princípios ativos testados, verificados no presente estudo, se comparados aos de Singh e Sanyal (1994), que encontraram cepas com baixa resistência, podem ser considerados preocupantes, uma vez que os consumidores de produtos de origem avícola correm o risco de infecções por microrganismos de alta resistência.

Tabela 4: Comportamento das cepas de Aeromonas hydrophila e Aeromonas caviae, isoladas de carcaças e cortes comerciais de frango, frente à ação de antimicrobianos

\begin{tabular}{|c|c|c|c|c|c|}
\hline \multirow[b]{2}{*}{ Antimicrobiano } & \multicolumn{2}{|c|}{ Aeromonas hydrophila } & \multicolumn{2}{|c|}{ Aeromonas caviae } & \multirow{2}{*}{$\begin{array}{l}\text { Total de } \\
\text { resistentes }\end{array}$} \\
\hline & Resistente & Sensivel & Resistente & Sensivel & \\
\hline Ampicilina & $29(100,0 \%)^{1}$ & - & $24(100 \%)$ & - & $53(100 \%)^{2}$ \\
\hline Polimixina B & $26(89,7 \%)$ & $03(10,3 \%)$ & $23(95,8 \%)$ & $01(4,2 \%)$ & $49(92,4 \%)$ \\
\hline Carbenicilina & $27(93,1 \%)$ & $02(6,9 \%)$ & $20(83,3 \%)$ & $04(16,7 \%)$ & $47(88,7 \%)$ \\
\hline Cefoxitina & $27(93,1 \%)$ & $02(6,9 \%)$ & $18(75,0 \%)$ & $06(25,0 \%)$ & $45(84,9 \%)$ \\
\hline Cefalotina & $25(86,2 \%)$ & $04(13,8 \%)$ & $19(79,2 \%)$ & $05(20,8 \%)$ & $44(83,0 \%)$ \\
\hline Sulfazotrim & $24(82,7 \%)$ & $05(17,3 \%)$ & $17(70,8 \%)$ & $07(29,2 \%)$ & $41(77,4 \%)$ \\
\hline Tetraciclina & $23(79,3 \%)$ & $06(20,7 \%)$ & $15(62,5 \%)$ & $09(37,5 \%)$ & $38(71,7 \%)$ \\
\hline Amicacina & $17(58,7 \%)$ & $12(41,3 \%)$ & $15(62,5 \%)$ & $09(37,5 \%)$ & $32(60,4 \%)$ \\
\hline Cloranfenicol & $19(65,5 \%)$ & $10(34,5 \%)$ & $12(50,0 \%)$ & $12(50,0 \%)$ & $31(58,5 \%)$ \\
\hline Netimicina & $16(55,2 \%)$ & $13(44,8 \%)$ & $12(50,0 \%)$ & $12(50,0 \%)$ & $28(52,8 \%)$ \\
\hline Tobramicina & $14(48,3 \%)$ & $15(51,7 \%)$ & $14(58,3 \%)$ & $10(41,7 \%)$ & $28(52,8 \%)$ \\
\hline Gentamicina & $15(51,8 \%)$ & $14(48,2 \%)$ & $12(50,0 \%)$ & $12(50,0 \%)$ & $27(50,9 \%)$ \\
\hline
\end{tabular}

1 Porcentagem em relação ao total de cepas da espécie

${ }^{2}$ Porcentagem em relação ao total das cepas estudadas
$\mathrm{Na}$ Tabela 5 verifica-se que o ágar dextrina-ampicilina mostrou-se mais eficiente que o ágar vermelho de fenolamido-ampicilina para o isolamento deAeromonas sp. a partir de amostras de frango, pois a partir do primeiro foram encontradas $33(30 \%)$ amostras positivas enquanto que o segundo revelou apenas $19(17,27 \%)$.

Tabela 5: Número de amostras positivas para bactérias do gênero Aeromonas, segundo o meio de cultura utilizado no plaqueamento seletivo, em relação aos tipos de amostras estudadas

\begin{tabular}{l|c|c|c}
\hline Tipo de amostra & $\begin{array}{c}\text { Total } \\
\text { Analisado }\end{array}$ & AVFAA $^{1}$ & ADA $^{2}$ \\
\hline Carcaça & 30 & $09(30,00)^{3}$ & $(50,00)$ \\
Peito & 50 & $02(4,00)$ & $14(28,00)$ \\
Coxa/sobrecoxa & 30 & $08(26,66)$ & $04(13,33)$ \\
\hline Total & 110 & $19(17,27)$ & $33(30,00)$ \\
\hline
\end{tabular}

1 Ágar vermelho de fenol-amido-ampicilina.

2 Ágar dextrina-ampicilina.

${ }^{3}$ Porcentagem em relação ao total de amostras analisadas.

A maioria dos estudos relativos à presença de aeromonas em alimentos utiliza mais de um meio de cultura na fase de isolamento seletivo, dada a inexistência de um meio $100 \%$ efetivo e tendo como objetivo melhorar a eficiência da técnica. Assim, vários trabalhos já foram realizados comparando meios de isolamento seletivo, podendo ser citados os de Abeyta Júnior et al. (1990), Chetti et al. (1993), Pin et al. (1994) e Rossi Júnior et al. (1996), que encontraram resultados bastante variáveis, sendo que os dois últimos utilizaram, dentre os meios testados, o ágar vermelho de fenol-amido-ampicilina.

Os resultados obtidos no presente estudo, onde um meio utilizado revelou $17,27 \%$ de amostras positivas e o outro $30 \%$, servem de alerta para a importância da utilização de mais de um meio de cultura na fase de isolamento de Aeromonassp. a partir de alimentos. Um dos meios que pode ser recomendado é o ágar dextrina-ampicilina (Havelaar e Vonk, 1988), pelo fácil reconhecimento das UFCs sugestivas do gênero e pela sua boa eficiência.

\section{Abstract}

Bacteria of the strain Aeromonas, have been incriminated as infections agents, both intestinal and extra-intestinal, being the animal-origin foods considered as important means of their transmissing into the human being, specially those products which get in touch with water during their obtaining or preparing. Therefore, due to limited information in national literature in respect to the aeromonas presence in poultry collected from commercial retailers, 30 samples of carcasses, 30 of legs/thighs and 50 chicken chests purchased in the city of Jaboticabal/SP were analized. The positivity for the strain was of $34.54 \%$ of the total analized samples, being that, in $29.09 \%$ of them (32) the strain A. hydrophila was isolated, and in $23.63 \%(26)$ the strain $A$. caviae. In relation to the products $50 \%$ of the samples of carcasses presented 
as positive, $28 \%$ of the chests and $30.00 \%$ of the legs and thighs, with minimal populations of $0.3 \times 10$ and maximum of $2.1 \times 10^{5} \mathrm{UFC} / \mathrm{gram}$ of sample. Around $80 \%$ of the isolated strains were resistent to polimixine, carbeniciline, cefoxitine and cefalotine and $49.0 \%$ to all the tested antimicrobians. In the selective isolation, the red agar phenol-starch-ampiciline revealed $17.27 \%$ of positive samples and the agar dextrine-ampiciline $30.0 \%$. The results showed a risk to the consumer, keeping in sight the significant number of samples with positive isolation and the high index of resistence verified.

Keywords: Aeromonas sp.; Aeromonas hydrophila; Aeromonas caviae; poultry; antimicrobians.

\section{Referências bibliográficas}

ABEYTA JÚNIOR C., KAYSNER, C.A., WEKELL, M.M., STOFF, R.F. Incidence of motile aeromonads from United States west coast shellfish growing estuaries. J Food Protn. 53, p. 849-855, 1990.

AGGER, W.A., Mccormick, J.D., Gurwith, M.J. Clinical and microbiological features of Aeromonas hydrophila associated diarrhea. J Clin Microbiol n. 21, p. 909-913, 1985.

BARNHART, H.M., PANCORBO, O.C., DREESEN, D.W., SHOTTS, E.B. Recovery of Aeromonas hydrophila from carcasses and processing water in a broiler processing operation. J Food Prot n. 52, p. 646-649, 1989.

BAUER, A.W., KIRBY, W.M., SHERRIS, J.C., TURCK, M. Antibiotic susceptibility testing by a standardized single disk method. Americ J Clin Path n. 45, p. 493-496, 1966.

BUCHANAN, R.L., PALUMBO, A.S. Aeromonas hydrophila and Aeromonas sobria as potential food poisoning species: a review. J Food Safety, n. 17, 15-29, 1985.

CHETTI, L. 1993. Isolation of Aeromonas spp. from chickens: their species and comparison of four selective media. Microbiol Alim Nutrit n. 11, p. 349-355.

CWIKOVÁ, O., HOVORKOVÁ, A., MRÁZ, O., STEINHAUSEROVÁ, I, MATYÁS, Z Aeromonads in slaughtered chickens: their species and pathogenic factors. Acta Vet. Bmo n. 62, p. 95-102, 1993.

FATHI, ShM, MOUSTAFA, S. Incidence of Aeromonas hydrophila in some selected frozen meat products in assiut city. Assiut Vet Med J. n. 26, p. 169-173, 1991.

FRICKER, C.R., TOMPSETT S. Aeromonas spp. in foods: A significant cause of food poisoning? Int J Food Microbiol $\mathrm{n}$. 9, p. 17-23, 1989.

GOBAT, P.F., JEMMI, T. Distribution of mesophilic Aeromonas species in raw and ready-to-eat fish and meat products in Switzerland. Int J Food Microbiol n. 20, p. 117-120, 1993.

HANDFIELD, M., SIMARD P., COUILLARD, M., LETARTE, R. Aeromonas hydrophila isolated from food and drinking water: hemagglutination, hemolysis and cytotoxicity for a human intestinal cell line (HT-29). Appl Environ Microbiol n. 62, p. 3459-3461, 1996.

HASSANI, L., IMZILN, B., BOUSSAID, A., GAUTHIER, M.J. Seasonal incidence of and antibiotic resistance among Aeromonas species isolated from domestic wastewater before and after treatment in stabilization ponds. Microb Ecol n. 23, p. 227-237, 1992.

HAVELAAR, A.H., VONK, M. The preparation of ampicillin dextrin agar for the enumeration of Aeromonas in water. Letters in Appl Microbiol n. 7, p. 169-171, 1988.

HÄNNINEN, M.L. Occurence of Aeromonas spp. in samples of ground meat and chicken. Int $J$ Food Microbiol n. 18, p. 339-342, 1993.
JINDAL, N., GARG, S.R., KUMAR, A. Comparison os Aeromonas spp. from human, livestock and poultry faeces. Isr $\mathrm{J}$ Vet Med n. 48, p. 80-83, 1993.

JOHNSTON, R.W., TOMPKIN, R.B. Meat and poultry products, p.821-835. In: VANDERZANT, C. e DF Splittstoesser, (eds.), Compendium of Methods for the Microbiological Examination of Foods, 3rd. ed., APHA, Washington, 1992.

KIPPERMAN, H., EPHROS, M., LAMBDIN, M., WHITE-ROGERS, K. Aeromonas hydrophila: A treatable cause of diarrhea. Pediatrics n. 73, p. 253-254, 1984.

KNOCHEL, S. Growth characteristics of motile Aeromonasspp. isolated from different environments. Int J Food Microbiol n. 10, p. 235-244, 1990.

KROVACEK, K., PETERZ, M, FARIS, A, MANSSON, I. Enterotoxigenicity and drug sensitivity of Aeromonas hydrophila isolated from well water in Sweden: a case study. Int J Food Microbiol n. 8, p. 149-154, 1989.

MAJEED, K.N., EGAN, AF, MAC ERA, I.C. Production of exotoxins by Aeromonas spp. at $5^{\circ} \mathrm{C}$. J Appl Bacterioln. 69, p. 332-337, 1990.

MERINO, S., RUBIRES, X., KNOCHEL, S., TOMÁS, J.M. Emerging pathogens: Aermonas spp. Int $J$ Food Microbiol n. 28, p. 157-168, 1995.

PALUMBO, A.S., BENCIVENGO, M.M., DEL CORRAL, F., WILLIAMS, A.C., BUCHANAN, R.L. Characterization of the Aeromonas hydrophila group isolated from retail foods of animal origin. J Clin Microbiol n. 27, p. 854-859, 1989.

MAXINO, F., WILLIAMS, A.C., BUCHANAN, R.L., THAYER, D.W. Starch-ampicillin agar for the quantitative detection of Aeromonas hydrophila. Appl Environ Microbiol n. 50, p. 1027-1030, 1985.

PICARD, B., Goullet P. Epidemiological complexity of hospital Aeromonas infections revealed by electrophoretic typing of esterases. Epidem Infect n. 98, p. 5-14, 1987.

PIN, C., MARIN, M.L., GARCIA, L.L., TORMO, J., CASAS, C. Comparison of different media for the isolation and enumeration of Aeromonas spp. in foods. Letters Appl Microbiol n. 18, p. 190-192, 1994.

POPOFF, M. Genus III. Aeromonas Kluyver and Van Niel. p. 545548. In: DRIEG, N. R. (ed.), Bergey's manual of systematic bacteriology, v. 1. Williams and Wilkins, Baltimoreme, 1984.

ROSSI JÚNIOR, O.D., NADER FILHO, A., AMARAL, LA, BARBOSA, AM Ocorrência de bactérias do gênero Aeromonas em carne bovina comercializada em Jaboticabal/SP e estudo comparativo entre meios de isolamento. ARS Vet n. 12, p. 64-73, 1996.

SINGH, D.V., SANYAL, SC Antibiotic resistance in clinical na environmental isolates of Aeromonas spp. J. Antimicrob Chemother n. 33, p. 368-369, 1994.

TOULE, G., MURPHY, O. A study of bacteria contaminating refrigerated cooked chicken: their spoilage potential and possible origin. J. Hyg Camb n. 81, p. 161-169, 1978. 